\title{
C1QBP regulates apoptosis of renal cell carcinoma via modulating xanthine dehydrogenase (XDH) mediated ROS generation
}

\section{Yiting Wang}

The Second Hospital of Tianjin Medical University; Tianjin Children's Hospital

\section{Qiqi Cui}

The Second Hospital of Tianjin Medical University

\section{Chao Wang}

The Second Hospital of Tianjin Medical University

\section{Shuang Liu}

The Second Hospital of Tianjin Medical University

\section{Runxuan Du}

The Second Hospital of Tianjin Medical University

\section{Shaoping Tian}

The Second Hospital of Tianjin Medical University

\section{Ruibing Chen}

Tianjin University

\section{Yuanjie Niu}

The Second Hospital of Tianjin Medical University

\section{Yong Wang}

The Second Hospital of Tianjin Medical University

\section{Dan Yue ( $\nabla$ danyue0705@sina.cn )}

Tianjin Medical University https://orcid.org/0000-0002-4220-4920

\section{Research}

Keywords: Renal cell carcinoma, C1QBP, XDH, ROS, apoptosis

Posted Date: October 29th, 2020

DOI: https://doi.org/10.21203/rs.3.rs-97866/v1

License: (1) (1) This work is licensed under a Creative Commons Attribution 4.0 International License. Read Full License 


\section{Abstract}

Background Complement component $1 \mathrm{Q}$ subcomponent binding protein (C1QBP) is a multifunctional protein and plays a vital role in the progression and metabolism of cancer. Our previous study has revealed that the low expression of C1QBP in renal cell carcinoma (RCC) and knockdown of C1QBP promotes the adhesion and invasion of RCC cells. However, its functions in the metabolism, oxidative stress and apoptosis of RCC cells have not yet been explored.

Methods Metabolomics assay was applied to investigate the role of C1QBP in RCC metabolism. C1QBP knockdown and overexpression cells were established via lentiviral infection and subjected to apoptosis and ROS assay in vitro. RNA stability assay and pre-mRNA detection were applied to characterize the mechanism of C1QBP regulating XDH transcription. In vivo, orthotopic tumor xenografts assay was performed to investigate the role of C1QBP progression.

Results Metabolomics investigations revealed that C1QBP dramatically diminished the hypoxanthine content in RCC cells. C1QBP promoted the mRNA and protein expression of hypoxanthine catabolic enzyme xanthine dehydrogenase $(\mathrm{XDH})$. Meanwhile, C1QBP regulated the expression of $X D H$ gene at the pre-transcriptional level by regulating XDH transcriptional stimulators IL-6, TNF-a and IFN- $y$. Moreover, the expression of C1QBP and XDH was lower in RCC tumors compared with the para-tumor normal tissues, and their down-regulation was associated with higher levels of Fuhrman grade. In RCC cells, C1QBP significantly increased produces reactive oxygen species (ROS) levels, apoptosis, and the expression of apoptotic proteins cleaved caspase-3 and bax/bcl2 via regulating XDH.

Conclusions C1QBP promotes the catabolism of hypoxanthine and elevates the apoptosis of RCC cells by modulating XDH-mediated ROS generation.

\section{Background}

Renal cell carcinoma (RCC) is one of the most common cancers in the urinary system, causing over 140,000 deaths worldwide each year [1]. Clear cell RCC (ccRCC) is the most common subtype among the malignant RCCs, accounting for $70-80 \%$ followed by papillary (15\%), chromophobe (4-5\%) and other less subtypes [2]. The onset of RCC is hidden and $16 \%$ of patients have metastasized at first diagnosis with a 5 -year survival of $9-12 \%$ [3]. Traditional chemotherapy and radiotherapy have limited effect in RCC, and molecular biomarkers are more targetable to guide treatment decisions. Recently, with the advent of immunotherapy and anti-angiogenesis therapy, the survival of RCC patients has been improved [4]. Despite the progresses of available therapeutic agents, significant challenges remain regarding patient selection and optimal therapeutic combinations.

Complement component $1 \mathrm{Q}$ subcomponent binding protein (C1QBP) also known as HABP1, SF2p32, $\mathrm{gC1qR}, \mathrm{p} 32$ is a highly conservative and multi-functional protein that ubiquitously distributes in the mitochondria matrix, cell membrane as well as in the secreted form, and it plays an important role in inflammation, infection, and cancer [5]. Studies have shown that C1QBP is overexpressed in malignant 
cells, e. g. breast, colon, and gastric cancers, in which it promotes various cellular processes, including cell survival, growth, apoptosis, and metastasis [5-8]. Interestingly, in our previous study, unlike other malignancies, C1QBP showed diminished expression in RCC compared with the corresponding paratumor tissues and suppressed adhesion and metastasis of RCC [9]. In addition, C1QBP as a mitochondria matrix protein maintains oxidative phosphorylation (OXPHOS) which produces reactive oxygen species (ROS) with the potential to damage cells and is essential for metabolism balance [10]. In addition, C1QBP regulates metabolism reprogramming to maintain the balance between OXPHOS and glycolysis in breast cancer [11]. However, the mechanism by which C1QBP regulates the metabolism and progression of RCC is still not completely clear.

Xanthine dehydrogenase (XDH), also known as xanthine oxidoreductase (XOR) and xanthine oxidase (XO) is the key enzyme for purine catabolism. XDH catalyzed the oxidation of hypoxanthine to xanthine and further catalyzed the formation of uric acid and ROS from xanthine [12]. XDH can also oxidize different endogenous metabolites and various exogenous substances to produce ROS, toxic substances and anticancer drugs [13]. Studies have shown that XDH-derived ROS on the one hand causes endothelial dysfunction, promotes inflammation and accelerates tumor growth, on the other hand, it can induce mutations or produce cytotoxic effects [14]. ROS accumulation can activate Fas and tumor necrosis factor receptor 1 (TNFR1) to bind to ligands, trigger caspase promoter caspase-8 activation and promote downstream bax expression, mitochondrial cytochrome $C$ release and caspase- 3 activation, leading to cell death [15].

Studies have shown that in liver, kidney and breast cancer tissues, the expression level of XDH is significantly lower than normal tissues, purine catabolism is severely impaired, and anabolic enzymes are increased [16-19]. This change gives tumor tissue a selective growth advantage through the rescue pathway of nucleic acid biosynthesis $[20,21]$. In serous ovarian cancer, the low expression of XDH is associated with more aggressive cancers and poor prognosis [22], indicating that XDH plays an important role in cancer progression and may become a risk factor or poor prognosis.

In present study, we investigated the roles of C1QBP in regulating purine metabolism and progression of RCC in vitro and in vivo. Our results showed that C1QBP regulates the catabolism of hypoxanthine and apoptosis in RCC cell by modulating XDH mediated ROS generation.

\section{Methods}

\section{Cell culture and establishment of stable cell lines}

Human RCC cell lines 786-O and ACHN and HEK-293T were obtained from American Type Culture Collection (ATCC, USA). The cells were grown in Dulbecco's Modified Eagle's Medium (DMEM) and Eagle's Minimum Essential Medium (MEM) (HAKATA, China) supplemented $10 \%$ fetal bovine serum (HAKATA, China) and $1 \times$ penicillin/streptomycin (Biological Industries, Israel) in a humidified $37^{\circ} \mathrm{C}$ incubator in the presence of $5 \% \mathrm{CO}_{2}$. 
To construct C1QBP overexpression and knocked-down stable cell lines, HEK-293T cells were cotransfected with lentiviral vectors (Promega, USA), including pLK0.1-Scr, pCDH-C1QBP, pCDH or pLK0.1shC1QBP and with packaging plasmid system consists of pSEV-REV, pMD 2.G and pRRE for $48 \mathrm{~h}$ using lipofectamine 2000 (Invitrogen, USA). The lentivirus supernatant was collected, centrifuged and filtered with $0.45 \mu \mathrm{m}$ filter. Then $786-0$ and $\mathrm{ACHN}$ cells were infected with the lentivirus supernatant respectively for $48 \mathrm{~h}$ and selected for 10 days with $2 \mu \mathrm{g} / \mathrm{ml}$ puromycin (Sangon Biotech, China) or $100 \mathrm{mg} / \mathrm{ml} \mathrm{G} 418$ (MDBio Inc, China), subsequently maintained with $0.5 \mu \mathrm{g} / \mathrm{ml}$ puromycin or $25 \mathrm{mg} / \mathrm{ml} \mathrm{G} 418$. The expression of C1QBP in the stable cell lines were determined using qRT-PCR and western blot.

\section{RNA extraction, reverse transcription and quantitative real-time PCR}

Total RNA extraction, reverse transcription and qRT-PCR analysis were performed as previously described [9]. The primers for qRT-PCR analysis were synthesized by HuaDa Gene. The primer sequences were showed in Table 1 (page 32). The expressions of target genes were normalized to GAPDH and the data were analyzed by the $2^{-\Delta \Delta C t}$ method.

\section{Western blot}

Proteins were extracted with SDS lysis buffer and $1 \times$ protease inhibitor cocktail (Roche, Germany) and the concentrations were measured by Enhanced BCA Protein assay kit (Thermo Fisher Scientific, USA). Proteins were separated by SDS-PAGE, then transferred onto a PVDF membrane (Millipore, USA), followed by blocking with $5 \%$ milk (BD, USA) in TBST (Tris-buffered saline with Tween-20) solution for $1.5 \mathrm{~h}$ at room temperature. Primary antibodies were incubated at $4{ }^{\circ} \mathrm{C}$ overnight. with corresponding primary antibodies to C1QBP (Abcam, USA, ab101267; 1:2000), XDH (Affinity, DF8111; 1:1000), caspase-3 (Abcam, ab184787; 1:2000), bcl2 (Abcam, ab182858; 1:2000), bax (Abcam, ab32503; 1:2000) and $\beta$-actin (Affinity Biosciences, T0022; 1:3000). In the next morning, after washing three times, the membranes were incubated with HRP-conjugated goat anti-rabbit IgG (Affinity, S0001; 1:5000) or goat anti-mouse IgG (Affinity, S0002; 1:4000) secondary antibodies for $1 \mathrm{~h}$ at room temperature, and developed by ECL Western Blotting Detection Reagents (Millipore, USA). The expressions of target proteins were normalized with $\beta$ actin.

\section{Metabolomics Relative-quantitative Analysis}

Well-growth and logarithmic 786-0 C1QBP overexpression and control cells $\varangle 1 \times 10^{7}$ \were washed twice with ice-cold PBS, and then washed cells with ice-cold saline (0.9\% sodium chloride solution) quickly, discarding the supernatant completely after each wash. Finally, cells were collected in a $1.5 \mathrm{ml}$ centrifuge tube with $1 \mathrm{ml}$ of methanol: acetonitrile: water $(2: 2: 1, \mathrm{v} / \mathrm{v})$ and stored at $-80{ }^{\circ} \mathrm{C}$ after quick freezing with liquid nitrogen.

The samples were thawed and mixed with $1 \mathrm{ml}$ cold methanol/acetonitrile/ $\mathrm{H}_{2} \mathrm{O} \otimes 2: 2: 1, \mathrm{v} / \mathrm{v} / \mathrm{v} \rrbracket$ and sonicated at low temperature ( $30 \mathrm{~min} /$ once, twice). Followed by centrifuged for $20 \mathrm{~min}\left(14000 \mathrm{~g}, 4{ }^{\circ} \mathrm{C}\right)$ and the supernatant was dried in a vacuum centrifuge. For LC-MS analysis, the samples were re-dissolved 
in $100 \mu$ lacetonitrile/water (1:1, v/v) and adequately vortexed, and then centrifuged $\left(14000 \mathrm{~g}, 4^{\circ} \mathrm{C}, 15\right.$ min). The supernatants were collected for LC-MS/MS analysis using an UHPLC (1290 Infinity LC, Agilent Technologies) coupled to triple quadrupole mass spectrometer.

MRM Analyzer software was used to extract chromatographic peak area and retention time. Detected metabolites in pooled samples with coefficient of variation (CV) less than $30 \%$ were denoted as reproducible measurements. In order to find the different expressed metabolites, statistical analyses between two sample groups were performed by calculating the fold changes and $p$ values of metabolites using Student t test, $p<0.05$, fold changes $>1.5$, were marked as the significantly changed metabolites between sample groups.

\section{Xanthine/hypoxanthine detection}

The level of xanthine/hypoxanthine was detected by using Xanthine/Hypoxanthine Colorimetric/Fluorometric Assay Kit (Bio Vision, USA,) in RCC cells. According to the manufacturer's protocol, cells $\left(1 \times 10^{6}\right)$ were lysed with $100 \mu$ ice cold Xanthine Assay Buffer for 10 min on ice, centrifuged at $12000 \mathrm{rpm}$ for 5 min followed by collecting the supernatant. The mixture of samples and reaction buffer were incubated for $30 \mathrm{~min}$ at room temperature, protected from light. Measure color at $\lambda=$ $570 \mathrm{~nm}$ using Microplate reader. Actually, calculating xanthine/hypoxanthine concentration (noml/ml) in the sample according to the standard curve.

\section{Human patients}

Human patient cohorts were used to explore the clinical importance of C1QBP and XDH expression in RCC. We collected 57 pairs of primary RCC tissues and corresponding para-carcinoma tissues. All RCC tissues were surgically removed and paraffin-embedded in the Second Hospital of Tianjin Medical University between January 2013 and December 2018 with the patients' consent and the approval from the ethical committee. Criteria for inclusion were that patients with pathological diagnosis of RCC and patients had undergone radical nephrectomy with no preoperative and postoperative adjuvant therapy. In addition, patient clinical pathological parameters were collected including gender, age, tumor size, tumor stage and Fuhrman grade.

\section{IHC staining}

Tissues were formalin-fixed and embedded in paraffin blocks and then cut into $5 \mu \mathrm{m}$ thickness sections. Tissue sections on glass slides were deparaffınized in xylene, and dehydrated in gradient ethanol, followed by blocking of endogenous peroxidase. Repaired antigens with boiling citrate buffer and blocked non-specific background staining with 5\% BSA (Solarbio, China). The tissue sections were incubated with primary antibody C1QBP (Santa Cruz Biotechnology, USA) or XDH overnight at $4{ }^{\circ} \mathrm{C}$. The second day, after washing with PBS, the slides were immunoblotted using Universal kit (mouse/rabbit polymer detection system) (ZSGB.BIO, China). Sections were developed by peroxidase substrate DAB 
Detection Kit (ZSGB.BIO, China) and then counterstained by hematoxylin. Finally, covered slides with neutral resin.

All immunostaining slides were scored by two independent researchers for the percentage and intensity of cells showing specific immunostaining signals. The score of percentage of positively stained cells was evaluated from 0 to 5 : $0,(0-1) \%$ cells positive; $1,(1-5) \%$ cells positive; $2,(6-10) \%$ cells positive; $3,(11-$ $20) \%$ cells positive; $4,(21-50) \%$ cells positive; and $5,(51-100) \%$ of cells positive. The score of immunostaining intensity as follows: 0 , no staining; 1 , yellow-brown staining; 2 , brown staining. The final score was calculated by adding the percentage and intensity scores and recorded as negative (0-3) and positive (4-7).

\section{RNA stability}

RCC cells were treated with $5 \mu \mathrm{g} / \mathrm{ml}$ actinomycin D (Med Chem Express, USA) at indicated time point 0 min, $15 \mathrm{~min}, 30 \mathrm{~min}, 45 \mathrm{~min}, 60 \mathrm{~min}$. The RNA was extracted using Trizol (Ambion, USA) and detected XDH mRNA levels by qRT-PCR.

\section{Reactive Oxygen Species (ROS) Assay}

The cells were detected with ROS Assay Kit (Beyotime Biotechnology, China) according to the manufacture's instruction. RCC cells were seeded at a density of $3 \times 10^{5}$ cells/well in 6-well plate and cultured overnight. The cells were treated with $10 \mu \mathrm{M} \mathrm{DCFH}-\mathrm{DA}$ and incubated at $37^{\circ} \mathrm{C}$ for $20 \mathrm{~min}$. Subsequently, washing cells three times to fully remove the DCFH-DA from liquid. And in positive group, cells were treated with $50 \mathrm{mg} / \mathrm{ml}$ Rosup for $20 \mathrm{~min}$. Afterwards, cells were collected and the levels of ROS were detected by fluorescent microplate reader at $\lambda=488 \mathrm{~nm} / 525 \mathrm{~nm}$ or detected via flow cytometry.

\section{Annexin V/PI apoptosis assay}

Renal cancer cells were detected with Annexin V-FITC apoptosis assay kit (Absin bioscience, China) according to the manufacture's instruction. The cells were harvested and counted and $5 \times 10^{5}$ cells were resuspended with $300 \mu \mathrm{l} 1 \times$ binding buffer. Subsequently, cells were stained with Annexin V-FITC and PI for $15 \mathrm{~min}$ in the dark, and detected by flow cytometer (BD FACS Verse, USA). The results were analyzed by Flow Jo vX.0.7 software (Flow Jo, USA).

\section{Small interfering RNA}

To investigate the role of XDH in RCC cells, XDH was knocked down by transient transfection of small interfering RNA (siRNA). Three independent XDH siRNA oligonucleotides and non-targeting sequences (negative controls) (Ribo Bio, China) were transfected into ACHN and 786-0 cells using Lipofectamine 2000 and $50 \mathrm{nmol} / \mathrm{L}$ of each siRNA according to the manufacture's protocol. The medium containing siRNAs were replaced 4-6 $\mathrm{h}$ after transfection. 
Total RNA was extracted $24 \mathrm{~h}$ after transfection and protein was extracted $48 \mathrm{~h}$ after transfection. The knockdown of gene expression was assessed by western blot and qRT-PCR. The sequences of XDH siRNA were listed in Table 2 (page 32).

\section{Xenograft tumor growth and metastasis}

Luciferase-labeled C1QBP overexpression and control of ACHN cells were stably constructed through lentivirus-mediated luciferase plasmids (ACHN-pCDH-luc and ACHN-pCDH-C1QBP-luc) and efficiencies of transduction including luminescence intensity and protein expression were verified using microplate reader and western blot, respectively. BALB/c Nude mice (Beijing Vital River Laboratory Animal Technology Co., Ltd.) ( $n=6$ each group, $6-8$ weeks old, male: female $=1: 1)$ were anesthetized and surgery was performed to expose the left kidney on the back of the mouse. Subsequently, tumor cells ( $1 \times$ $10^{6}$ cells, mixed with Matrigel, 1:1) were injected under the renal capsule and incisions were sutured. Eight weeks later, mice were intraperitoneally injected with luciferin (30 $\mu \mathrm{g}$ per mouse), and detected bioluminescence signals of primary tumors and metastasis in liver and lung by the live IVIS imaging system (Perkin Elmer, USA). Mice were sacrificed, and tumors of the left kidney, liver and lungs were removed. The weight of primary tumor of mice were calculated by using minus the weight of corresponding right kidney. Primary RCC tumors, livers, and lungs were fixed in formalin, embedded in paraffin and then cut into sections. The proteins expressions in sections of primary RCC tumor were observed by IHC staining with antibodies of C1QBP, XDH, caspase-3, bax and bcl2, and metastases of lungs and livers of mice were examined with HE staining. Actually, the difference between two groups were analyzed using $t$ test.

\section{Xenograft tumor growth and metastasis}

Liver and lung tissues of BALB/c mice were formalin-fixed and embedded in paraffin and then cut into 5 $\mu \mathrm{m}$ thickness sections. Tissue sections on glass slides were deparaffinized in xylene, and hydrated in gradient ethanol from high to low concentrations and soaked in distilled water, followed by staining with hematoxylin and eosin. Next, the sections were dehydrated in gradient ethanol from low to high concentrations ethanol and hyalinized in xylene then covered glasses with neutral resin. Sections were observed the metastases under the microscope and took pictures.

\section{Statistical analysis}

Statistical analysis was performed by GraphPad Prism 7.0 and SPSS 21.0 software. All data were represented as the mean $\pm S D$. Differences between two groups were used t- test. Correlation analysis between C1QBP and XDH expressions was analyzed with Spearman test. $\chi^{2}$ test was used for analysis of the correlations between protein expression and clinicopathologic features. $P<0.05$ was considered as statistically significant.

\section{Results}




\section{C1QBP regulates the levels of hypoxanthine in RCC}

First, we established C1QBP overexpression and knocked-down stable RCC cell lines 786-0 and ACHN, and then verified the expression of C1QBP by western blot and real-time PCR (Fig. 1a and b). To further explore the roles of C1QBP in the regulation of cellular metabolism, we examined the abundances of 200 key metabolites in C1QBP overexpression 786-0 cells and the corresponding control cells. Analytes were quantified by multiple reaction monitoring (MRM) in both positive or negative ion modes which were listed in Supplemental Table 1 in both controls and C1QBP expression 786-0 cells $(n=6)$. Analytes were determined by electrospray ionization (ESI) MRM in both the positive or negative ion modes respectively. The results showed that the overexpression of C1QBP led to significant abundance changes of 17 metabolites in 786-0 cells (Table 3, page 33). Intriguingly, hypoxanthine was reduced by $80 \%$ in C1QBP overexpressing cells compared with the control group $\triangle$ Fig. 1c). Next, we verified the impact of C1QBP upregulation on the level of hypoxanthine in 786-0 and $\mathrm{ACHN}$ cells. Consistent with the metabolomics result, C1QBP overexpression significantly decreased the level of hypoxanthine (Fig. 1e). The above data indicate that C1QBP involves in the regulation of RCC cell metabolism and reduces the level of hypoxanthine.

\section{C1QBP regulates the expression of XDH in RCC}

$\mathrm{XDH}$ is a key enzyme in purine metabolism which oxidizes hypoxanthine to xanthine and catalyzes the conversion of xanthine to uric acid [12]. Previous research has shown that high tumoral XDH expression predicted poor prognosis in patients with lung adenocarcinoma [23]. We investigated whether C1QBP regulated the hypoxanthine level via XDH. The expression of XDH was detected by western blot and realtime PCR. C1QBP knockdown decreased the mRNA and protein expression of XDH in 786-0 and ACHN cells (Fig. 2a and b). In addition, to investigate the expression of C1QBP and XDH in RCC tissues, 30 pairs of ccRCC tissues and their corresponding adjacent normal kidney tissues were examined by western blot. The results showed that C1QBP and XDH expressions were significantly $(P=0.015, P<0.0001)$ decreased in the ccRCC tissues when compared with adjacent non-cancerous kidney tissues (Fig. 2c and d). In addition, statistical analysis showed that there was a positive correlation between the expression of C1QBP and XDH in ccRCC tissues (Table 4, page 33). Furthermore, 57 pairs of ccRCC tissues were subjected to immunohistochemical $(\mathrm{IHC})$ staining and representative pictures were shown in Fig. 2e. Consistent with the western blot results $\triangle \mathrm{IHC}$ showed that both $\mathrm{C} 1 \mathrm{QBP}$ and XDH were down-regulated in cCRCC tissues, and further statistical correlation analysis revealed that C1QBP positively correlated with $\mathrm{XDH}(r=0.628, P<0.001)$ (Table 5, page 34).

The association of C1QBP and XDH expressions with RCC clinicopathologic features was also analyzed. The results showed that the expression of C1QBP and XDH in RCC patients was both significantly $(P<$ $0.05)$ related to patients' Fuhrman grade. In addition, C1QBP expression was related to TNM staging of ccRCC patients. However, their expression was not significantly associated with other clinicopathological characteristics, including gender, age and tumor size (Table 6, page 34). Overall, the findings indicate that C1QBP positively regulates XDH expression in RCC. 


\section{C1QBP modulates XDH mRNA at pre-translational level}

A previous study showed that C1QBP as RNA-binding protein is involved in RNA splicing [24]. To investigate whether C1QBP may regulate the splicing of the XDH RNA, we examined the level of XDH premRNA by quantitative real-time PCR using primers targeting junction. However, the results indicated that depletion of C1QBP significantly inhibited the XDH pre-mRNA levels (Fig. 3a), while the result of C1QBP overexpression is opposite (Fig. 3b), suggesting that the regulation of $\mathrm{XDH}$ gene expression by C1QBP occurs at the pre-transcriptional level. The expression of XDH is affected by many factors, including inflammatory cytokines, hormones, growth factors and stimuli etc [14]. Inflammatory cytokines TNF-a, IL6, IL-1 $\beta$ and IFN- $\gamma$ have been reported to promote the transcription of XDH [25]. To examine whether C1QBP affects transcriptional stimulators of XDH, we measured the mRNA levels of TNF-a, IL6, IL-1 $\beta$ and IFN- $y$. Results showed that C1QBP positively regulated TNF- $a$, IL-6 and IFN- $y$ mRNA expression in RCC cells, among which the change of TNF-a is the most obvious (Fig. $3 \mathrm{c}$ and d). In addition, we also investigated the role of C1QBP on the stability of XDH mRNA. The results showed that the deprivation of C1QBP didn't affect RCC cells XDH mRNA stability (Fig. 3e). The results indicate that C1QBP regulates the expression of XDH at the pre-transcriptional level by regulating XDH transcriptional stimulators IL-6, TNF$a$ and IFN-Y.

\section{C1QBP modulates ROS level and apoptosis in RCC}

$\mathrm{XDH}$ involves in the oxidative purine metabolism by catalyzing hypoxanthine to produce xanthine and ROS [26,27], and the activation of XDH generates oxidative stress [28]. We examined fluorescent probe DCF (2',7'-dichlorofluorescein, DCF) fluorescence intensity represented as ROS level in RCC cells, the results showed that DCF fluorescence was significantly decreased in C1QBP knockdown ACHN and 786$O$ cell lines (Fig. 3a, left panel). In contrast, C1QBP overexpression increased DCF fluorescence intensity (Fig. 3a, right panel). The findings indicate that C1QBP promotes ROS generation in RCC cells.

ROS plays critical role in cell apoptosis, so we investigated whether C1QBP impact apoptosis of RCC cells (Fig. 3b and d). We found that C1QBP downregulation inhibited cell apoptosis (Fig. 3c) and C1QBP overexpression promoted cell apoptosis (Fig. 3e). Meanwhile, we examined apoptosis associated protein expression by western blot in $\mathrm{ACHN}$ and 786-0 cells. The results demonstrated that C1QBP enhanced the expression of apoptotic protein bax and cleaved-caspase-3 and diminished anti-apoptotic protein bcl2 (Fig. 3f). These results indicate that C1QBP modulates ROS generation and apoptosis of RCC cells.

\section{C1QBP regulates ROS and apoptosis of RCC cells via XDH.}

To investigate whether C1QBP regulated ROS level and cell apoptosis via modulating the expression of $\mathrm{XDH}$, we did the rescue assay. As showed in Fig. 4a and b, the third siRNA (si-XDH-3) was the most effective in XDH knockdown. In C1QBP overexpression ACHN and 786-0 cells, transfection with XDH siRNA partially inhibited C1QBP induced ROS (Fig. 4c and d) and cell apoptosis (Fig. 4e and) increasing. And, the change was consistent with the expressions of the bax and cleaved-caspase-3. Corresponding deprivation of XDH partially restored the expression of the anti-apoptotic protein bcl2 (Fig. $4 \mathrm{~g}$ ). The 
findings revealed that $\mathrm{C} 1 \mathrm{QBP}$ regulated ROS production, cell apoptosis and the expression of apoptosis related proteins via regulating XDH in RCC cells.

\section{C1QBP overexpression suppresses RCC tumor growth, metastasis and the expressions of XDH and apoptosis-related proteins in vivo}

To determine the role of C1QBP in RCC process in vivo, we constructed orthotopic tumor xenograft model with implantation of stable C1QBP overexpressing and control ACHN cells with luciferase into the left kidney capsule of BALB/c nude mice. After 8 weeks, mice were intraperitoneally injected with luciferin, and live imaging was performed to detect the bioluminescence intensity of the primary tumor and liver and lung metastases. The results showed that mice in the C1QBP overexpression group had lower bioluminescence intensity comparing with mice in the control group (Fig. 5a and b). Next, primary tumors were isolated from mice in these two groups, and the tumor weight was lower in C1QBP overexpression group (Fig. 5C), suggesting that C1QBP plays an important role in RCC tumor growth in vivo. In addition, the role of C1QBP on metastasis of RCC was confirmed. Compared to control group, the bioluminescence intensity of liver (Fig. 5d and e) and lung (Fig. 5h and i) was lower in C1QBP overexpression mice group. In order to further observe the effect of C1QBP on liver and lung metastasis in vivo, we performed HE staining. Consistently, the results demonstrated there was significantly fewer liver (Fig. $5 f$ and g) and lung (Fig. 5j and k) metastatic nodules in C1QBP overexpression group than control group. Moreover, slices of primary renal tumor were performed IHC staining. There was an increasing expression of XDH in C1QBP overexpression group (Fig. 5I), suggesting that C1QBP could enhance XDH expression. Additionally, in C1QBP overexpression group, increased expressions of caspase- 3 and bax and decreased bcl2 expression were detected (Fig. $5 \mathrm{l}$ ). The data indicate that C1QBP regulates growth and metastasis of RCC in vivo.

\section{Discussion}

RCC often involves systemic metastases and the recurrence rate of RCC patients after surgery is still as high as 30\% [29]. It is well known that RCC is not sensitive to both chemotherapy and radiotherapy, and the serious adverse reactions caused by immunotherapy and the side effects and resistance of drugs in targeted therapy present challenges for the treatment of RCC [30]. Therefore, to develop new targeted anticancer drugs, in-depth research of the molecular basis of RCC progression is of great significance for improving the survival of patients with RCC. In previous study, we found that C1QBP suppressed progression of RCC, while the mechanism remains unclear. In this study, we found that C1QBP regulated RCC cell purine catabolic metabolism via positively regulating XDH mRNA and protein expression in RCC cells. Furthermore, we determined that the expressions of protein C1QBP and XDH were decreased in cCRCC comparing with corresponding adjacent normal kidney tissues. And both of C1QBP and XDH proteins were associated with higher level of Fuhrman grade. Mechanically, C1QBP stimulated the expression of IL-6, TNF- $a$ and IFN- $\gamma$ which promoted XDH transcription. Functionally, we demonstrated that C1QBP enhanced the ROS levels and apoptosis in RCC cells. We also reached consistent conclusions that C1QBP inhibited tumor growth and metastasis and increased expressions of XDH and pro-apoptotic 
related proteins in mice orthotopic tumor xenografts model. Remarkably, we revealed that C1QBP promoted the production of ROS, cell apoptosis and catabolic metabolism of hypoxanthine in RCC via regulating $\mathrm{XDH}$.

Given the numerous important roles in multiple disease, C1QBP has now become a potential target for the development of monoclonal antibody-based and/or small molecule-based therapies [5]. However, little is known about the molecular mechanism of C1QBP in metabolism and progression of RCC. C1QBP plays critical roles in mitochondria protein synthesis, maintenance of oxidative phosphorylation, and tumor metabolism reprograming, suggesting the importance of C1QBP in metabolism [5,11]. In present study, we applied metabolomics to analyze differential metabolites in C1QBP overexpression RCC cells among 200 main metabolites. Due to the low content of some metabolites囚only 109 metabolites were detected of which 17 metabolites changed significantly, involving in amino acid, glucose metabolism and nucleotide metabolism. The results showed that the content of hypoxanthine changed most significantly so we focused on hypoxanthine. Meanwhile, we observed that the nucleotide metabolites in the upstream of hypoxanthine metabolism remained unchanged (Supplemental table 2). Therefore, we demonstrated C1QBP may be involved in RCC cell hypoxanthine catabolism.

$\mathrm{XDH}$ is strictly modulated at the transcriptional and post-translational levels and highly expressed in the breast, liver, intestine, kidney and vascular endothelial cells while lowly expressed in the lung and skin [31]. Studies have showed that the expression of XDH may be positively associated with a worse outcome in gastric and lung cancer [32,33]. However, XDH expression decreased in RCC and negatively associated with a high malignity grade and a worse prognosis [14]. Consistently, we get the similar conclusion that the lower XDH expression in RCC tissue is associated with higher Fuhrman grade, and there is a positive correlation between C1QBP and XDH in RCC tissues. In addition, we found that C1QBP positively regulated XDH mRNA and protein expression in RCC cells. A previous study has shown that C1QBP, as a splicing factor, can control RNA splicing by isolating necessary RNA splicing factors into inhibitory complexes [34]. While, C1QBP increased RCC cell XDH pre-mRNA levels, suggesting that C1QBP regulated XDH gene expression at pre-transcriptional level or inhibited RNA splicing. We further demonstrated that C1QBP promoted XDH transcription via stimulating the mRNAs of XDH transcription stimulating factors IL-6, TNF- $a$ and IFN- - . These results revealed that C1QBP promoted hypoxanthine catabolism via promoting pre-transcriptional level of XDH.

Oxidative stress, resulting from an imbalance between pro-oxidants and antioxidants, has been recognized as a consequence of aging directly related to lifespan [35]. ROS-driven oxidative stress has been recognized as a critical inducer of cancer cell death in response to therapeutic agents [36]. Evidence from study has shown that ROS promoted apoptosis by activating caspase 3 in RCC cells [37]. And C1QBP caused excessive production of ROS and apoptosis in fibroblasts [38]. In present study, we revealed that C1QBP overexpression promoted ROS levels and apoptosis of RCC. Oxidative stress caused by XDH [28] promoted prostate cancer cell-specific apoptosis [39]. We further explored the effect of XDH in RCC and confirmed that the increasing ROS and apoptosis caused by C1QBP overexpression was 
partially restored via silencing $\mathrm{XDH}$. Therefore, $\mathrm{XDH}$ is critical for C1QBP regulated ROS and apoptosis in RCC.

The apoptosis inhibitory factor bcl 2 is the key regulator of apoptosis. Anti-apoptotic bcl 2 and proapoptotic bax can form ion channels in mitochondria, resulting in changes in mitochondrial permeability (macropore formation) and the release of apoptotic protein activator cytochrome c. And the occurrence of apoptosis is accompanied by the release of cytochrome $\mathrm{c}$ from mitochondria, followed by the activation of caspase, which eventually leads to apoptosis [15]. The accumulation of ROS leads to an increase in the ratio of bax/bcl2 and promotes the activation of caspase-3 to promote apoptosis [40]. Our study showed that C1QBP enhanced the activation of caspase-3, the expression of bax and inhibited the expression of bcl2 via XDH in vivo and in vitro. Our findings may imply that C1QBP affects cell apoptosis by regulating the expression of bax/bcl 2 and the activation of caspase- 3 which were modulating by $\mathrm{XDH}$ mediated ROS generation and eventually suppresses RCC progression.

In summary, the expressions of C1QBP and XDH are significantly lower in RCC compared to adjacent normal tissues, and associated with high Fuhrman grade. C1QBP regulates catabolism of hypoxanthine via $\mathrm{XDH}$. And the regulation of $\mathrm{XDH}$ transcription by C1QBP is proved to be accomplished by stimulating the expressions of XDH transcription factors IL-6, TNF- $a$ and IFN- $\gamma$. In addition, C1QBP plays an important role in promoting apoptosis via regulating XDH mediated ROS generation in RCC. Our findings suggest that C1QBP is a novel mediator of RCC tumor progression and targeting C1QBP/XDH provides a potential treatment strategy for RCC. However, the exact mechanism of regulation of XDH expression by C1QBP still needs further investigation.

\section{Conclusions}

We have clarified that C1QBP promotes RCC cell hypoxanthine catabolism via up-regulating XDH, and apoptosis by modulating XDH mediated ROS generation and pro-apoptotic proteins caspase-3 and bax/bcl2, then affects tumor progression. In addition, C1QBP promotes XDH gene expression at the pretranscriptional level by stimulating the gene expressions of XDH transcriptional stimulators IL-6, TNF-a and IFN-Y.

\section{Abbreviations}

C1QBP: Complement component $1 \mathrm{Q}$ subcomponent binding protein; ccRCC: Clear cell renal cell carcinoma; $\mathrm{CO}_{2}$ : Carbon dioxide; DDW: double distilled water; IHC: Immunohistochemistry; MRM: multiple reaction monitoring; $\mathrm{NaCl}$ : Sodium chloride; OXPHOS: oxidative phosphorylation; PCR: Polymerase chain reaction; ROS: reactive oxygen species; RCC: Renal cell carcinoma; shRNA: Short hairpin RNA; siRNA: Small interfering RNA; TNFR1: tumor necrosis factor receptor 1; TNM: Tumor, node, metastasis; XDH: xanthine dehydrogenase; XOR: xanthine oxidoreductase; XO: xanthine oxidase;

\section{Declarations}


Ethics approval and consent to participate: The study was approved by the Institutional Review Board of Tianjin Medical University and was carried out in accordance with the principles outlined in the Declaration of Helsinki. Informed consent was obtained from each patient used in this study.

Consent for publication: Not applicable.

Availability of data and material: The datasets used and/or analyzed during the current study are available from the corresponding author on reasonable request.

Conflict of interest: The authors declare that they have no competing interests.

Funding: This work was supported by the National Natural Science Foundation of China [grant numbers 81772945, 81872078, and 21974094], the Natural Science Foundation of Tianjin [grant number 18JCYBJC26700 and 18JCYBJC25200], the Young Elite Scientists Sponsorship Program [grant number TJSQNTJ-2017-10], and National Training Program of innovation and Entrepreneurship for undergraduates [grant number 202010062044].

Authors' contributions: Yong Wang, and Dan Yue conceived the study and designed the experiments. Yiting Wang, Qiqi Cui, and Chao Wang performed in vitro experiments; Yiting Wang analyzed data; Yiting Wang, Qiqi Cui, Chao Wang Shuang Liu, Xuanrun Du, and Shaoping Tian performed immunohistochemical staining and in vivo experiment; Yiting Wang, Qiqi Cui, and Chao Wang organized clinical samples; Yiting Wang, Yong Wang, and Dan Yue wrote the manuscript. Dan Yue, Yong Wang, Ruibing Chen, and Yuanjie Niu modified the manuscript.

\section{Acknowledgments:}

We are grateful to Shanghai Applied Protein Technology for assistance on metabolomic LC-MS/MS analysis.

\section{References}

1. Ferlay J, Soerjomataram I, Dikshit R, Eser S, Mathers C, Rebelo M, et al. Cancer incidence and mortality worldwide: sources, methods and major patterns in GLOBOCAN 2012. Int J Cancer. 2015;136(5):E359-86.

2. Nandagopal L, Sonpavde GP, Agarwal N. Investigational MET inhibitors to treat Renal cell carcinoma. Expert Opin Investig Drugs. 2019;28(10):851-60.

3. Sun X, Gan L, Na A, Ge L, Chen B, Liu J. Combination with Stereotactic Body Radiotherapy Offers a Promising Strategy to Overcome Resistance to Immunotherapy in Advanced Renal Cell Cancer. J Oncol. 2019;2019:1483406.

4. Motzer RJ, Escudier B, Gannon A, Figlin RA. Sunitinib: Ten Years of Successful Clinical Use and Study in Advanced Renal Cell Carcinoma. Oncologist. 2017;22(1):41-52. 
5. Ghebrehiwet B, Geisbrecht BV, Xu X, Savitt AG, Peerschke EIB. The C1q Receptors: Focus on gC1qR/p33 (C1qBP, p32, HABP-1)(1). Semin Immunol. 2019; 45:101338.

6. Rubinstein DB, Stortchevoi A, Boosalis M, Ashfaq R, Ghebrehiwet B, Peerschke El, et al. Receptor for the globular heads of $\mathrm{C} 1 \mathrm{q}$ ( $\mathrm{gC} 1 \mathrm{q}-\mathrm{R}, \mathrm{p} 33$, hyaluronan-binding protein) is preferentially expressed by adenocarcinoma cells. Int J Cancer. 2004;110(5):741-50.

7. Peerschke El, Brandwijk RJ, Dembitzer FR, Kinoshita Y, Ghebrehiwet B. Soluble gC1qR in Blood and Body Fluids: Examination in a Pancreatic Cancer Patient Cohort. Int J Cancer Res Mol Mech. 2015;1(3).

8. Xiao K, Wang Y, Chang Z, Lao Y, Chang DC. p32, a novel binding partner of Mcl-1, positively regulates mitochondrial $\mathrm{Ca}(2+)$ uptake and apoptosis. Biochem Biophys Res Commun. 2014;451(2):322-8.

9. Wang Y, Fu D, Su J, Chen Y, Qi C, Sun Y, et al. C1QBP suppresses cell adhesion and metastasis of renal carcinoma cells. Sci Rep. 2017;7(1):999.

10. Muta T, Kang D, Kitajima S, Fujiwara T, Hamasaki N. p32 protein, a splicing factor 2-associated protein, is localized in mitochondrial matrix and is functionally important in maintaining oxidative phosphorylation. J Biol Chem. 1997;272(39):24363-70.

11. Fogal V, Richardson AD, Karmali PP, Scheffler IE, Smith JW, Ruoslahti E. Mitochondrial p32 protein is a critical regulator of tumor metabolism via maintenance of oxidative phosphorylation. Mol Cell Biol. 2010;30(6):1303-18.

12. Xu P, Huecksteadt TP, Hoidal JR. Molecular cloning and characterization of the human xanthine dehydrogenase gene (XDH). Genomics. 1996;34(2):173-80.

13. Pritsos CA, Gustafson DL. Xanthine dehydrogenase and its role in cancer chemotherapy. Oncol Res. 1994;6(10-11):477-81.

14. Battelli MG, Polito L, Bortolotti M, Bolognesi A. Xanthine oxidoreductase in cancer: more than a differentiation marker. Cancer Med. 2016;5(3):546-57.

15. Fulda S, Debatin KM. Extrinsic versus intrinsic apoptosis pathways in anticancer chemotherapy. Oncogene. 2006;25(34):4798-811.

16. Chen GL, Ye T, Chen HL, Zhao ZY, Tang WQ, Wang LS, et al. Xanthine dehydrogenase downregulation promotes TGFbeta signaling and cancer stem cell-related gene expression in hepatocellular carcinoma. Oncogenesis. 2017;6(9):e382.

17. De Lamirande G, Allard C, Cantero A. Purine-metabolizing enzymes in normal rat liver and Novikoff hepatoma. Cancer Res. 1958;18(8 Part 1):952-8.

18. Yin J, Ren W, Huang X, Deng J, Li T, Yin Y. Potential Mechanisms Connecting Purine Metabolism and Cancer Therapy. Front Immunol. 2018;9:1697.

19. Reid E, Lewin I. Adenosine deaminase, nucleoside phorphorylase and xanthine oxidase in liver tumours. Br J Cancer. 1957;11(3):494-8.

20. Prajda N, Morris HP, Weber G. Imbalance of purine metabolism in hepatomas of different growth rates as expressed in behavior of xanthine oxidase (EC 1.2.3.2). Cancer Res. 1976;36(12):4639-46. 
21. Weber G, Hager JC, Lui MS, Prajda N, Tzeng DY, Jackson RC, et al. Biochemical programs of slowly and rapidly growing human colon carcinoma xenografts. Cancer Res. 1981;41(3):854-9.

22. Linder N, Butzow R, Lassus H, Lundin M, Lundin J. Decreased xanthine oxidoreductase (XOR) is associated with a worse prognosis in patients with serous ovarian carcinoma. Gynecol Oncol. 2012;124(2):311-8.

23. Konno H, Minamiya Y, Saito H, Imai K, Kawaharada Y, Motoyama S, et al. Acquired xanthine dehydrogenase expression shortens survival in patients with resected adenocarcinoma of lung. Tumour Biol. 2012;33(5):1727-32.

24. Saha SK, Kim KE, Islam SMR, Cho SG, Gil M. Systematic Multiomics Analysis of Alterations in C1QBP mRNA Expression and Relevance for Clinical Outcomes in Cancers. J Clin Med. 2019;8(4).

25. KD P, TP H, JR H. Xanthine dehydrogenase and xanthine oxidase activity and gene expression in renal epithelial cells. Cytokine and steroid regulation. Journal of immunology (Baltimore, Md: 1950). 1994;153(4):1789-97.

26. Berry CE, Hare JM. Xanthine oxidoreductase and cardiovascular disease: molecular mechanisms and pathophysiological implications. J Physiol. 2004;555(Pt 3):589-606.

27. Wu B, Hao Y, Shi J, Geng N, Li T, Chen Y, et al. Association between xanthine dehydrogenase tag single nucleotide polymorphisms and essential hypertension. Mol Med Rep. 2015;12(4):5685-90.

28. Abrigo J, Elorza AA, Riedel CA, Vilos C, Simon F, Cabrera D, et al. Role of Oxidative Stress as Key Regulator of Muscle Wasting during Cachexia. Oxid Med Cell Longev. 2018; 2018:2063179.

29. Patel C, Ahmed A, Ellsworth P. Renal cell carcinoma: a reappraisal. Urol Nurs. 2012;32(4):182-90; quiz 91.

30. Kotecha RR, Motzer RJ, Voss MH. Towards individualized therapy for metastatic renal cell carcinoma. Nat Rev Clin Oncol. 2019;16(10):621-33.

31. Chung HY, Baek BS, Song SH, Kim MS, Huh JI, Shim KH, et al. Xanthine dehydrogenase/xanthine oxidase and oxidative stress. Age (Omaha). 1997;20(3):127-40.

32. Tavassoly I, Hu Y, Zhao S, Mariottini C, Boran A, Chen Y, et al. Genomic signatures defining responsiveness to allopurinol and combination therapy for lung cancer identified by systems therapeutics analyses. Mol Oncol. 2019;13(8):1725-43.

33. Liu H, Zhu H, Shi W, Lin Y, Ma G, Tao G, et al. Genetic variants in XDH are associated with prognosis for gastric cancer in a Chinese population. Gene. 2018; 663:196-202.

34. Petersen-Mahrt SK, Estmer C, Ohrmalm C, Matthews DA, Russell WC, Akusjarvi G. The splicing factorassociated protein, p32, regulates RNA splicing by inhibiting ASF/SF2 RNA binding and phosphorylation. EMBO J. 1999;18(4):1014-24.

35. Kregel KC, Zhang HJ. An integrated view of oxidative stress in aging: basic mechanisms, functional effects, and pathological considerations. Am J Physiol Regul Integr Comp Physiol. 2007;292(1):R1836. 
36. Li K, Gao B, Li J, Chen H, Li Y, Wei Y, et al. ZNF32 protects against oxidative stress-induced apoptosis by modulating C1QBP transcription. Oncotarget. 2015;6(35):38107-26.

37. Lopes TZ, de Moraes FR, Tedesco AC, Arni RK, Rahal P, Calmon MF. Berberine associated photodynamic therapy promotes autophagy and apoptosis via ROS generation in renal carcinoma cells. Biomed Pharmacother. 2020; 123:109794.

38. Saha P, Chowdhury AR, Dutta S, Chatterjee S, Ghosh I, Datta K. Autophagic vacuolation induced by excess ROS generation in HABP1/p32/gC1qR overexpressing fibroblasts and its reversal by polymeric hyaluronan. PLoS One. 2013;8(10): e78131.

39. Xu H, Li C, Mozziconacci O, Zhu R, Xu Y, Tang Y, et al. Xanthine oxidase-mediated oxidative stress promotes cancer cell-specific apoptosis. Free Radic Biol Med. 2019; 139:70-9.

40. Kelekar A, Thompson CB. Bcl-2-family proteins: the role of the BH3 domain in apoptosis. Trends Cell Biol. 1998;8(8):324-30.

\section{Tables}

Table 1 Primers sequences

\begin{tabular}{ll}
\hline Genes & \multicolumn{1}{c}{ Primers sequences (5' to 3') } \\
\hline GAPDH & F: GCCGTCTATGCGGCTTGT \\
& R: TGGAAGGGGTTCCCTGAGTT \\
$X D H$ & F: TGCACCACCAACTGCTTAGC \\
& R: GGCATGGACTGTGGTCATGAG \\
C1QBP & F: AGTGCGGAAAGTTGCCGGGGA \\
& R: GAGCTCCACCAGCTCATCTGC \\
pre-XDH & F: CCTAGCAACCCAGCAGACTCC \\
& R: TCCACAGCCGAGCTTGGTTC \\
\hline
\end{tabular}

Table 2 The sequences of siRNAs 


\begin{tabular}{ll}
\hline Name & Target sequence \\
\hline si-XDH-1 & GACCTGAGCTGAAGATCGA \\
si-XDH-2 & GAACTACCAGCCATTATCA \\
si-XDH-3 & GCATCGTCATGAGTATGTA \\
\hline
\end{tabular}

Table 317 significantly differentially expressed metabolites after C1QBP overexpression in 786-O cells

\begin{tabular}{lcc}
\hline Metabolite name & \multicolumn{2}{l}{ Fold change $P$-value } \\
\hline Hypoxanthine & 0.200 & 0.010 \\
N-Acetyl-D-glucosamine & 0.665 & 0.024 \\
L-Methionine & 0.704 & 0.006 \\
L-Leucine & 0.723 & 0.041 \\
L-Tyrosine & 0.764 & 0.005 \\
\hline gamma-L-Glutamyl-L-valine & 0.768 & 0.043 \\
3-Methyluridine & 0.867 & 0.018 \\
Glycine & 0.877 & 0.022 \\
L-Proline & 0.945 & 0.035 \\
L-O-Phosphoserine & 1.221 & 0.033 \\
Nicotinamide & 1.721 & 0.042 \\
\hline Acetyl-DL-Leucine & 1.744 & 0.006 \\
N-Carbamoyl-L-aspartic acid & 1.926 & 0.047 \\
\hline Dihydroxy-acetone-phosphate 2.218 & 0.008 \\
\hline Cytidine & 2.311 & 0.033 \\
\hline Deoxycytidine & 2.450 & 0.012 \\
\hline D-Glucose 6-phosphate & 2.840 & 0.014 \\
\hline
\end{tabular}

Table 4 Clinical relevance of $\mathrm{C} 1 \mathrm{QBP}$ and $\mathrm{XDH}$ in RCC by western blot 


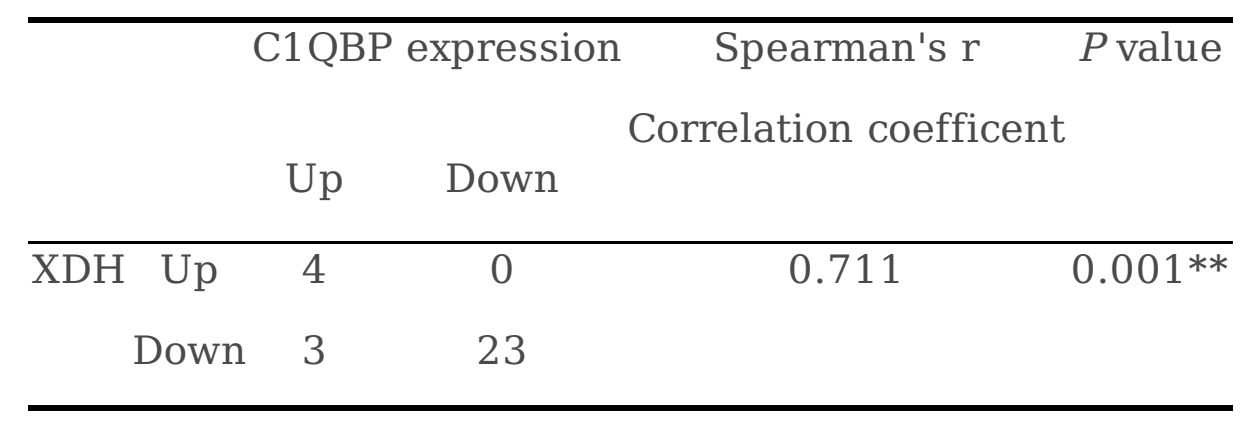

Note: The expressions of C1QBP and XDH were quantified and compared between RCC tumor $(\mathrm{T})$ and paired adjacent normal tissue $(\mathrm{N})$. The relative ratio $\mathrm{T} / \mathrm{N}>1$ was defined as "Up", and $\mathrm{T} / \mathrm{N}<1$ was defined as "Down".

Table 5 Clinical relevance of C1QBP and XDH in RCC by IHC

\begin{tabular}{cccccc}
\hline & & C1QBP expression & Spearman's r Correlation coefficent & $P$ value \\
\cline { 3 - 4 } & & positive & negative & & \\
\hline XDH & positive & 14 & 8 & 0.628 & $0.000^{* * * *}$ \\
& negative & 2 & 33 & & \\
\hline
\end{tabular}

Note: IHC detected the expression of protein C1QBP and XDH in 57 ccRCC tissues. Spearman's correlation coefficient analysis, $* * *, P<0.001$ difference is statistically significant.

Table 6 Expression of C1QBP and XDH in RCC tissues 


\begin{tabular}{|c|c|c|c|c|c|c|c|c|}
\hline & & \multicolumn{3}{|c|}{ XDH expression } & \multirow[t]{2}{*}{$P$ value } & \multicolumn{2}{|c|}{ C1QBP expression } & \multirow[t]{2}{*}{$P$ value } \\
\hline & & $\mathrm{N}$ & - & + & & - & + & \\
\hline \multirow[t]{2}{*}{ Gender } & Male & 40 & 26 & 14 & 0.658 & 27 & 13 & 0.342 \\
\hline & Female & 17 & 10 & 7 & & 14 & 3 & \\
\hline \multirow[t]{2}{*}{ Age } & $<65$ & 30 & 19 & 11 & 0.977 & 23 & 7 & 0.402 \\
\hline & $\geq 65$ & 27 & 17 & 10 & & 18 & 9 & \\
\hline \multirow[t]{3}{*}{ Tumor size } & $<4$ & 12 & 8 & 4 & 0.886 & 10 & 2 & 0.613 \\
\hline & $\geq 4, \leq 7$ & 16 & 10 & 6 & & 11 & 5 & \\
\hline & $>7$ & 29 & 17 & 12 & & 20 & 9 & \\
\hline \multirow[t]{2}{*}{ TNM stage } & $\mathrm{T} 1-2$ & 40 & 25 & 15 & 0.794 & 27 & 13 & 0.044 \\
\hline & T3-4 & 17 & 10 & 7 & & 16 & 1 & \\
\hline \multirow[t]{2}{*}{ Fuhrman grade } & I,II & 31 & 15 & 16 & $0.028 *$ & 17 & 15 & $0.026 *$ \\
\hline & III,IV & 26 & 20 & 6 & & 15 & 2 & \\
\hline \multirow[t]{2}{*}{ Location } & Carcinorma & 57 & 35 & 22 & $0.000 * * *$ & 41 & 16 & $0.000 * * *$ \\
\hline & $\begin{array}{c}\text { para- } \\
\text { carcinorma }\end{array}$ & 57 & 4 & 53 & & 13 & 44 & \\
\hline
\end{tabular}

Note: IHC staining results (+): positive, (-): negative.

Figures 
a

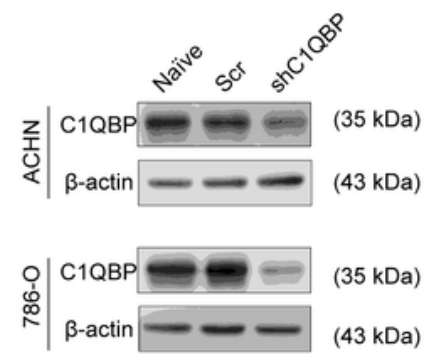

b

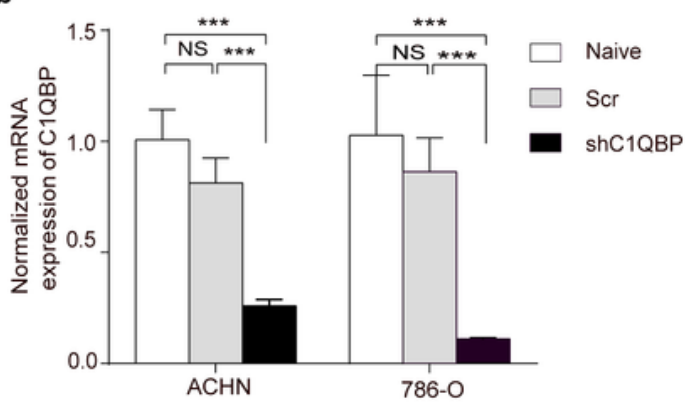

c

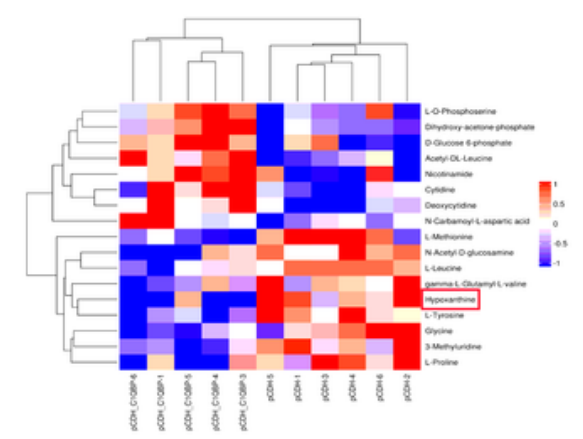

e

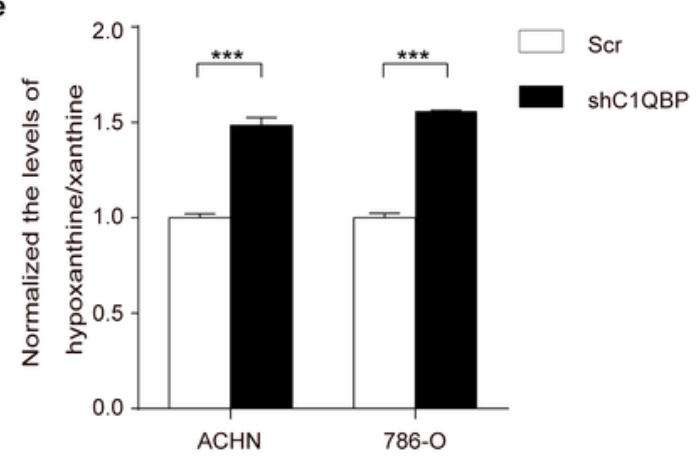

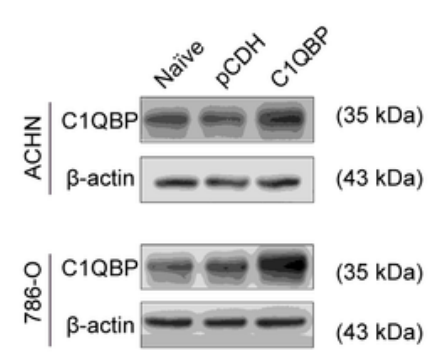

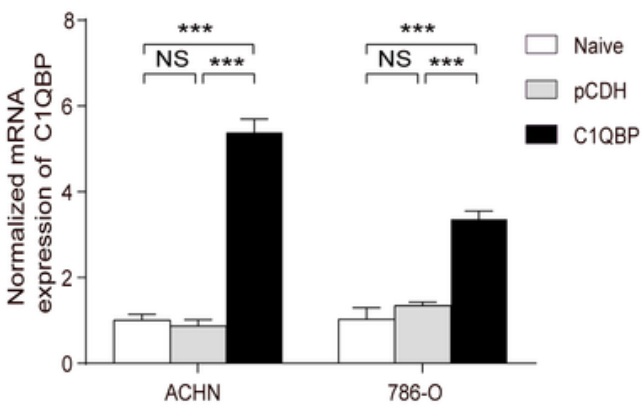

d
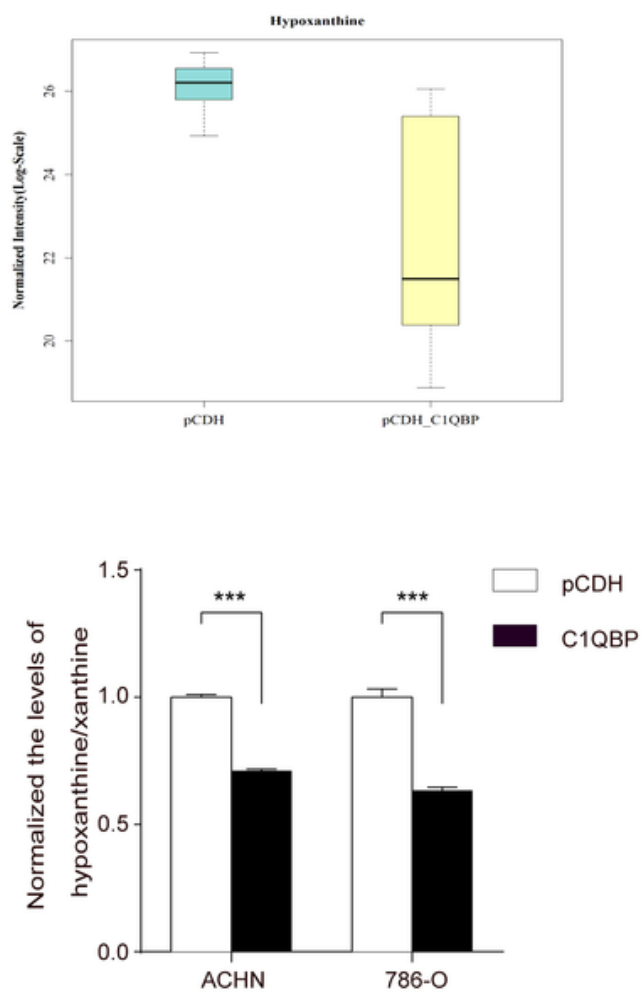

\section{Figure 1}

C1QBP regulates the levels of hypoxanthine in RCC. ACHN and 786-0 cells were transduced with lentivirus-mediated Scr control, shC1QBP or pCDH control, pCDH-C1QBP, and the expression of C1QBP was examined by western blot (a) and real time PCR (b). (c) According to the manufacturer's instructions 107 cells each group were collected and froze with liquid nitrogen. Metabonomic techniques were used to detect the differences of 200 major metabolites involved in cell metabolism in RCC 786-0 cells control 
group (pCDH) and C1QBP overexpression group (pCDH-C1QBP), $\mathrm{n}=6$. (d) Among the 17 differential metabolites, the change of hypoxanthine was the most significant, and decreased by $80 \%$ in $786-0$ cells with overexpression of C1QBP compared with the control group, $P=0.01, n=6$. (e) The level of hypoxanthine was examined by assay kit in ACHN and 786-0 cells with C1QBP knockdown (left panel) and overexpression (right panel), $\mathrm{P}<0.001$ by $\mathrm{t}$ test, data were presented as mean $\pm \mathrm{SD}$ from three independent repeats. Naïve: untreated; Scr: down-expression empty plasmid control; shC1QBP: C1QBP knockdown; pCDH: overexpression empty plasmid control; C1QBP/pCDH-C1QBP: C1QBP overexpression. Statistically significant differences were indicated: ${ }^{\star \star}, \mathrm{P}<0.001$. NS: no significant difference. 

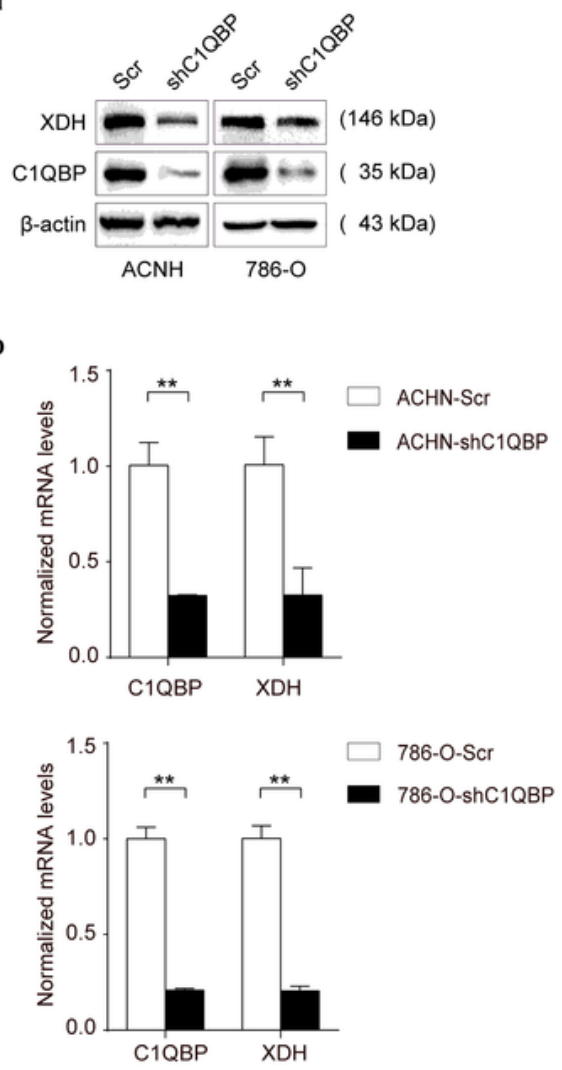
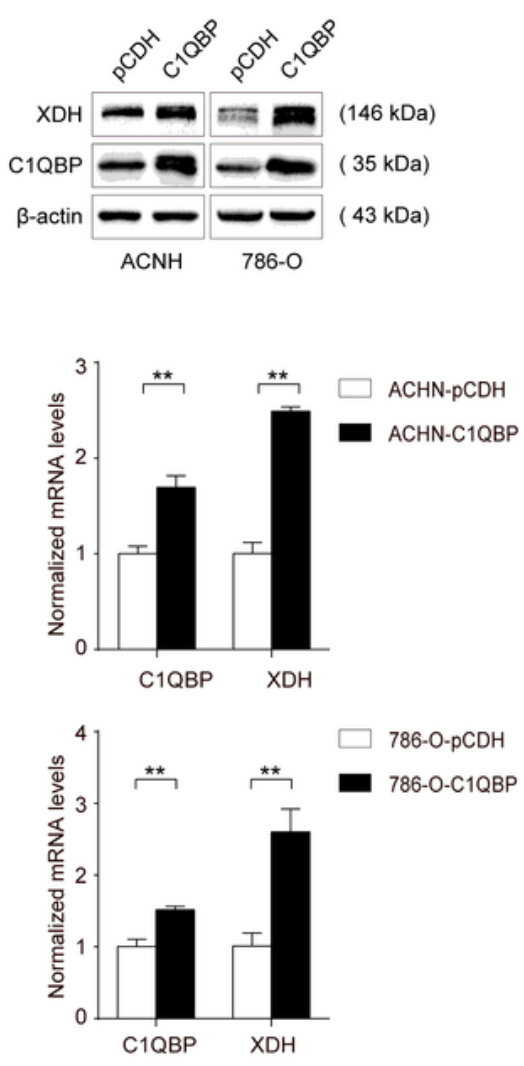

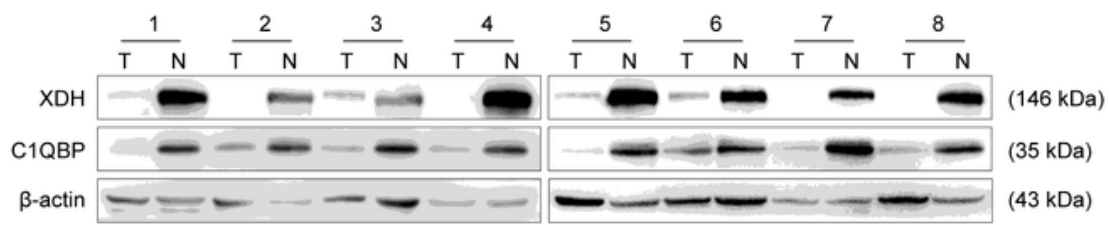

d

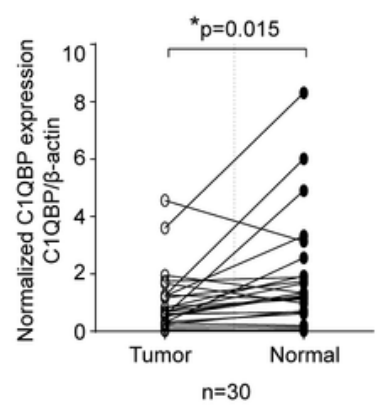

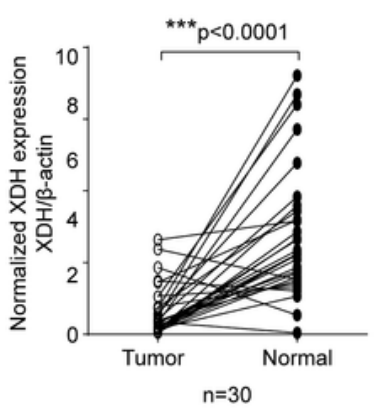

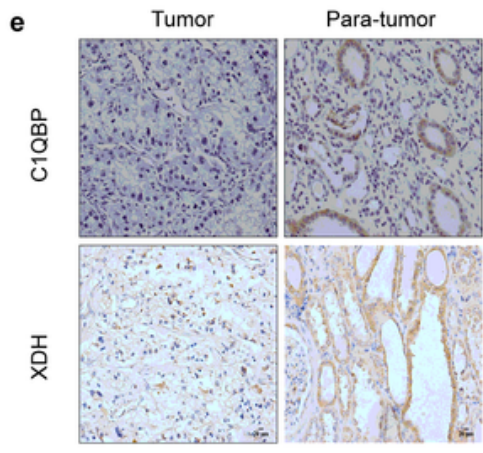

$50 \overline{\mu \mathrm{m}}$

\section{Figure 2}

C1QBP regulates the expression of XDH in RCC. (a) Western blot detected the expressions of XDH in ACHN and 786-0 cells with stable C1QBP knockdown (left panel) and C1QBP overexpression (right panel). (b) The mRNA levels of XDH were examined by real time PCR in ACHN (upper panel) and 786-0 (lower panel) cells with stable C1QBP knockdown (left panel) and C1QBP overexpression (right panel). The expressions of protein C1QBP and XDH were detected by western blot in both tumor (T) and adjacent 
normal tissues $(\mathrm{N})$ of 30 primary RCC patients. (c) Representative 8 pairs of western blot images were shown. (d) The expressions of C1QBP (left panel) and XDH (right panel) were normalized with $\beta$-actin and quantified by Image $\mathrm{J}$ software in 30 primary RCC patients. The analysis was used paired t-test, $\mathrm{P}=$ $0.015, P<0.0001$, respectively, $n=30$. (e) C1QBP and XDH expressions were examined by immunohistochemistry staining in a study cohort with 57 pairs of RCC (Tumor) and adjacent normal kidney tissues (Para-tumor). Representative pictures were shown, and brown signals indicated positive staining. Scale bar $=50 \mu \mathrm{m}$. Statistically significant differences were indicated: ${ }^{*}, P<0.05, * \star, P<0.01$ and $\star \star \star, P<0.001$. 

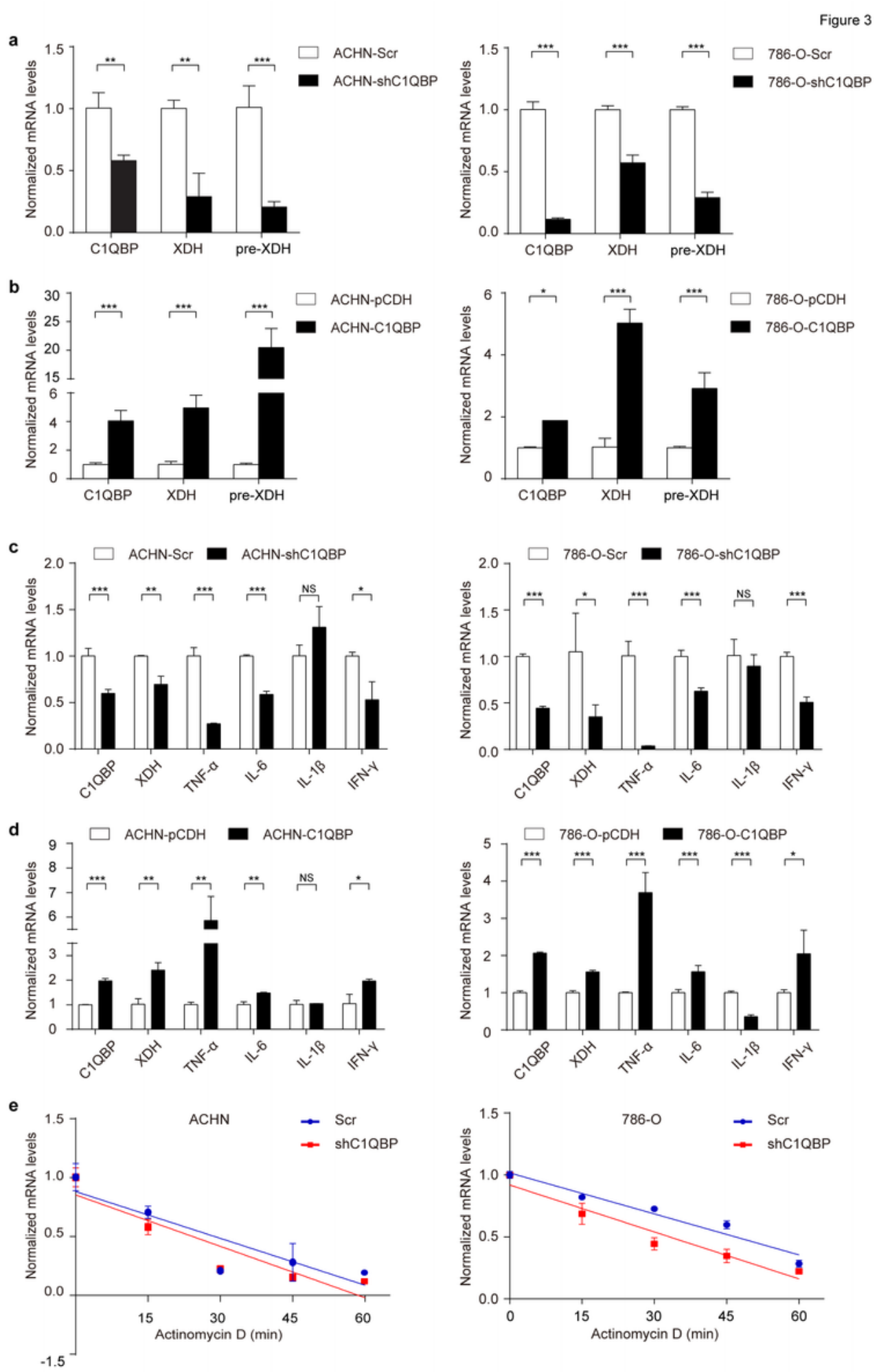

Figure 3

C1QBP modulates XDH mRNA at pre-translational level. The levels of XDH pre-mRNA were examined by quantitative real-time PCR in control groups and C1QBP knockdown or overexpression group of ACHN (a) and 786-O (b) cells. The mRNA levels of TNF- $a$, IL6, IL-1 $\beta$ and IFN- $\gamma$ were examined by quantitative realtime PCR in control groups and C1QBP knockdown or overexpression group of ACHN (c) and 786-0 (d) cells. (e) Control and C1QBP overexpressed ACHN and 786-0 cells were treated with $5 \mu \mathrm{g} / \mathrm{ml}$ actinomycin 
D at indicated time point $0 \mathrm{~min}, 15 \mathrm{~min}, 30 \mathrm{~min}, 45 \mathrm{~min}, 60 \mathrm{~min}$. The RNA was extracted and detected XDH mRNA levels using quantitative real-time PCR technology. Statistically significant differences were indicated: ${ }^{*}, \mathrm{P}<0.05, * *, \mathrm{P}<0.01$ and $* * *, \mathrm{P}<0.001$. NS: no significant difference.

a

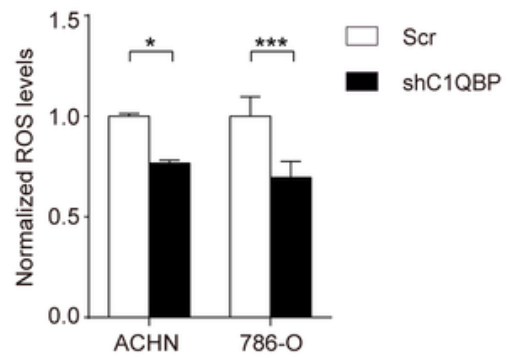

Figure 4

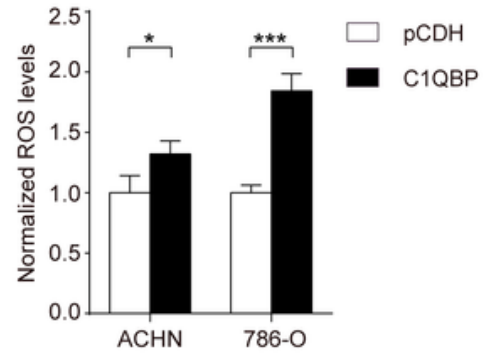

b

$\bar{a}$

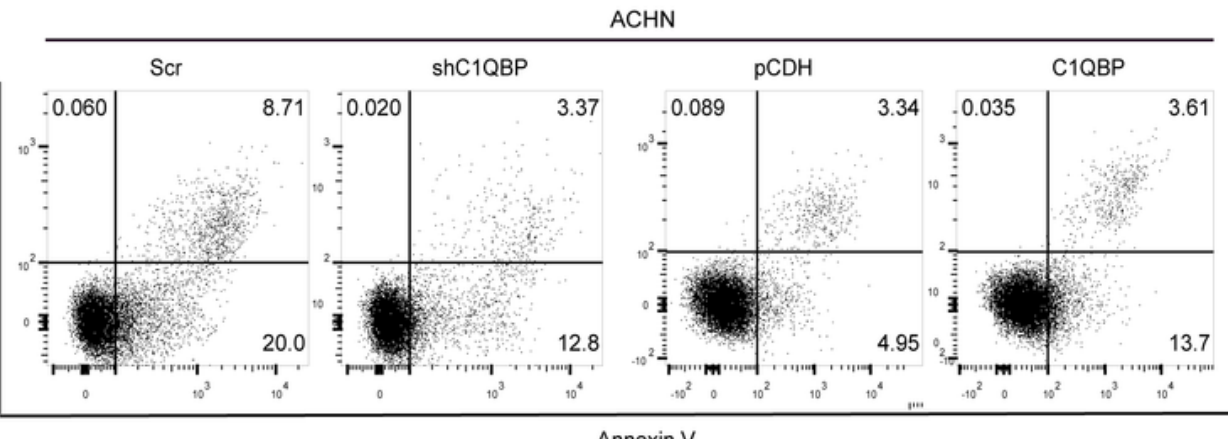

d

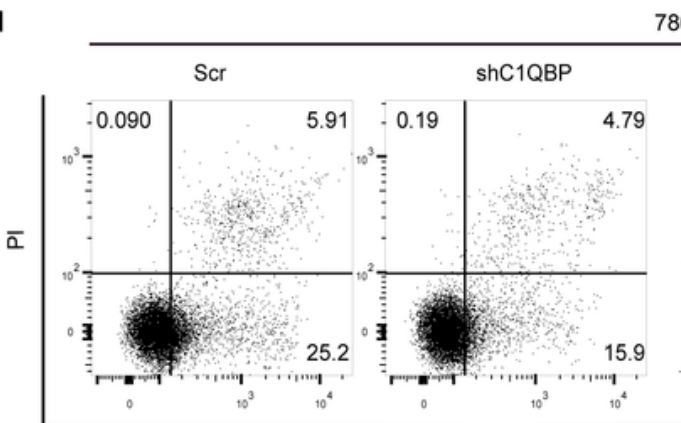

c
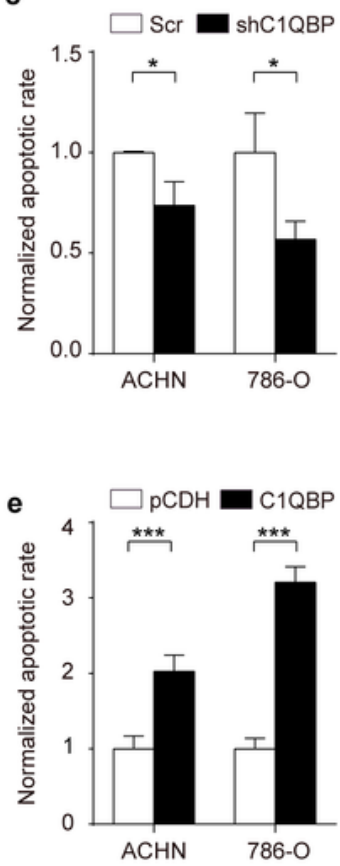

Annexin V
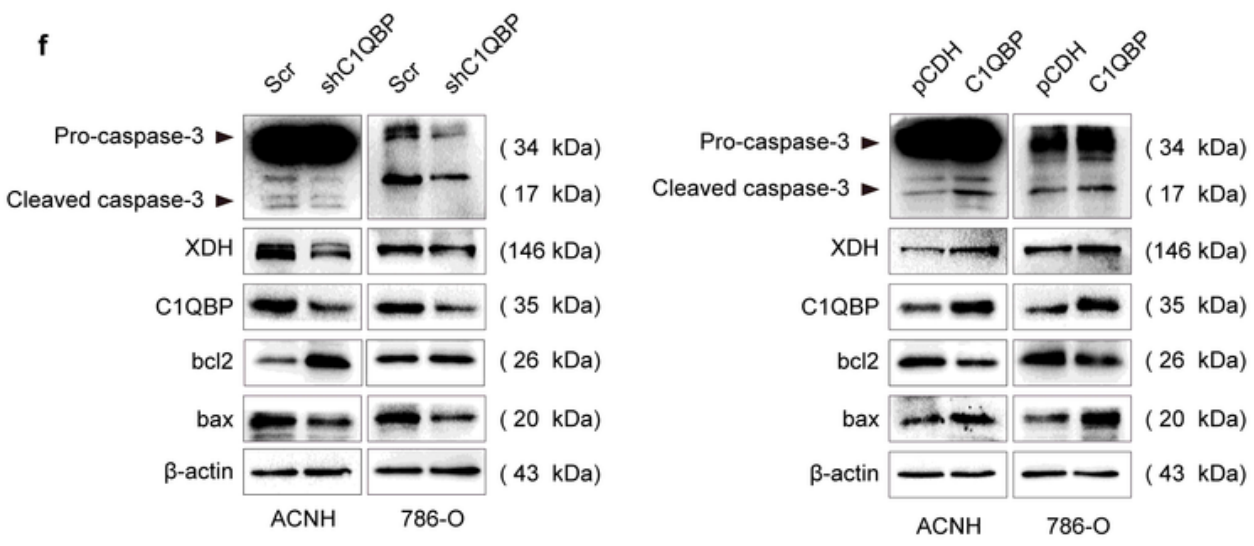

Figure 4

C1QBP is critical for R0S levels and apoptosis of RCC cells. (a) In ACHN and 786-0 RCC cells, ROS levels were examined and quantified by detecting fluorescence intensity of DCF and normalizing to control. Left 
panel: control and C1QBP knockdown group, right panel: control and C1QBP overexpression group. In ACHN and 786-O RCC cells, percentage of annexin V-positive cells was determined by flow cytometry (b and d). Quantitative analysis of apoptotic rate of cells with C1QBP knockdown (c) and C1QBP overexpression (e) was performed by normalizing to corresponding control group. (f) The expressions of C1QBP, XDH, cleaved-caspase-3, bcl2 and bax were examined by western blot in ACHN and 786-O cells with controls and C1QBP knockdown and overexpression. Data were presented as mean \pm SD by $t$ test, $n$ $=3 ; *, \mathrm{P}<0.05, * *, \mathrm{P}<0.01$, and $* \star *, \mathrm{P}<0.001$ compared to the control (Scr or $\mathrm{PCDH})$.

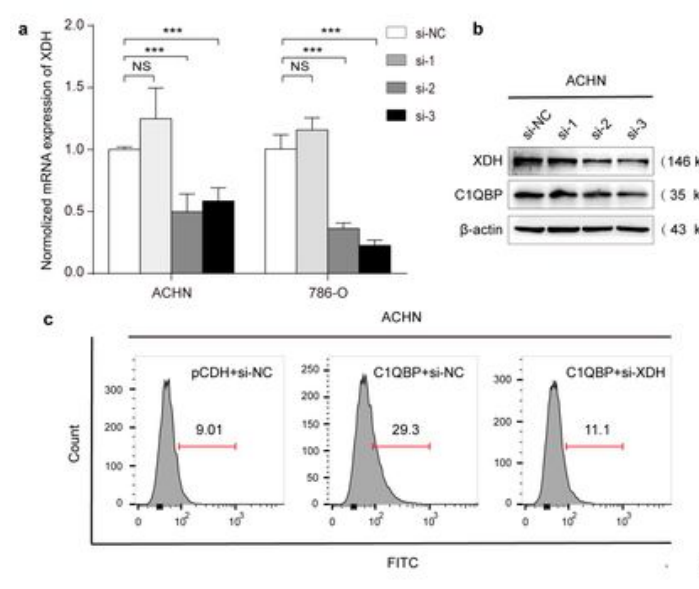
Figure 5
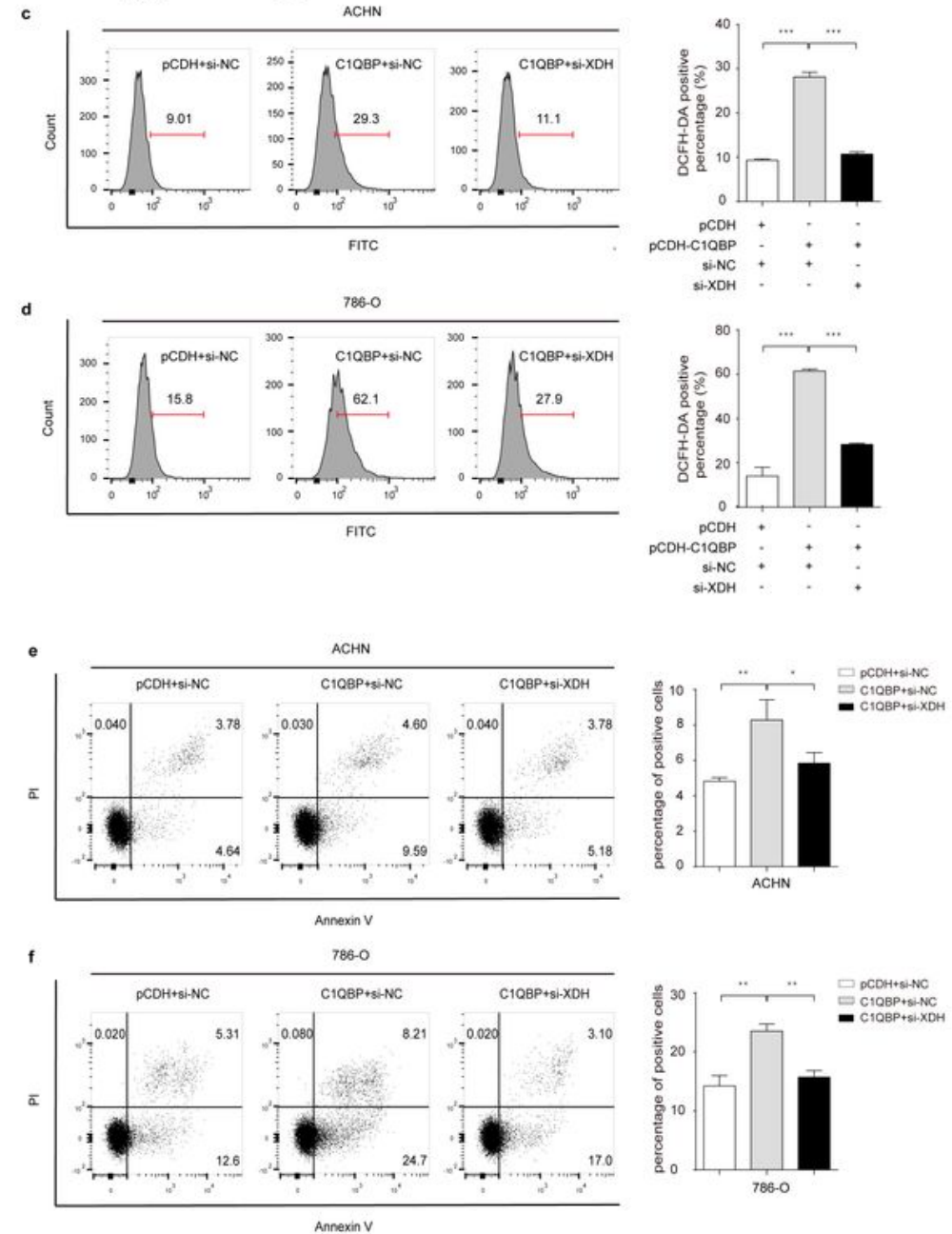

g
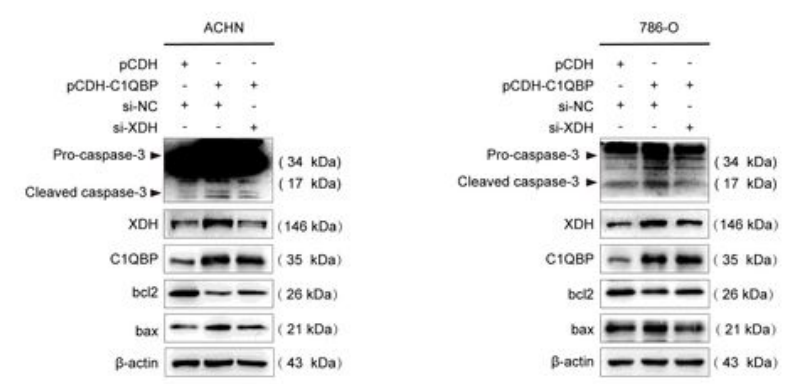


\section{Figure 5}

XDH is critical for C1QBP-regulated ROS production and apoptosis of RCC. XDH knockdown by using three independent XDH siRNAs (si-XDH-1, si-XDH-2, si-XDH-3) in ACHN and 786-0 cells were evidenced by real time RCR (a) and western blot (b). ACHN and786-0 cells were transfected with pCDH + si-NC, pCDH-C1QBP + si-NC, pCDH -C1QBP + si-XDH and then ROS levels (c and d) and apoptosis (e and f) were examined by flow cytometry, and (g) the expression levels of C1QBP, XDH, cleaved-caspase-3, bcl2 and bax were examined by western blot. $\beta$-actin was used as an internal control. Data were presented as mean \pm SD by $t$ test. Statistically significant differences were indicated: $*, P<0.05, * \star, P<0.01$ and $* \star \star, P$ $<0.001$. NS: no significant difference. 
a
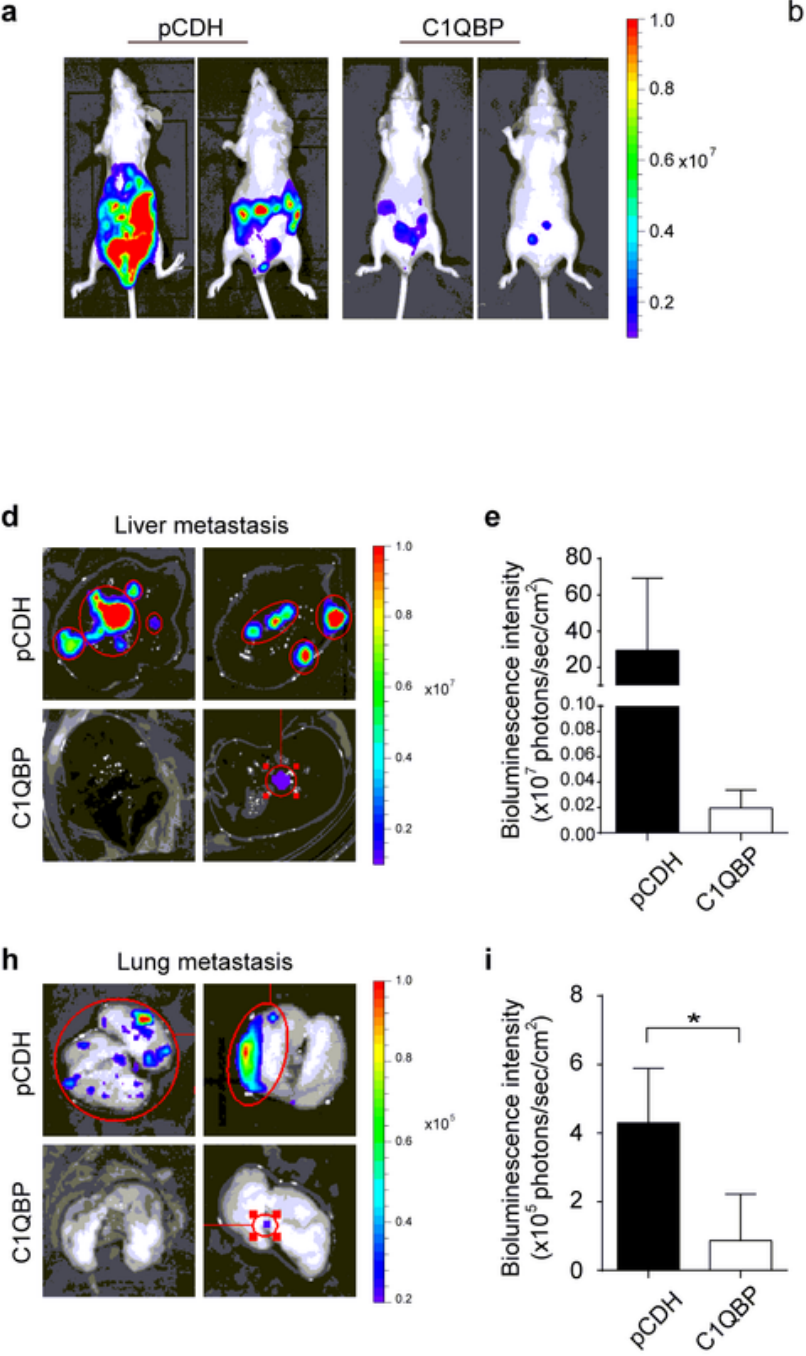

$b$
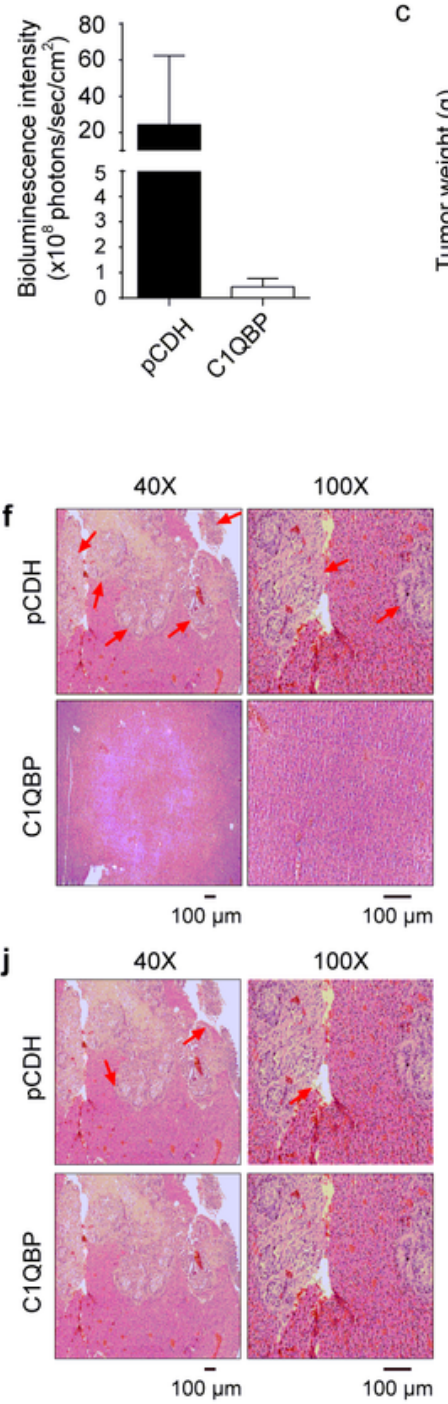

c

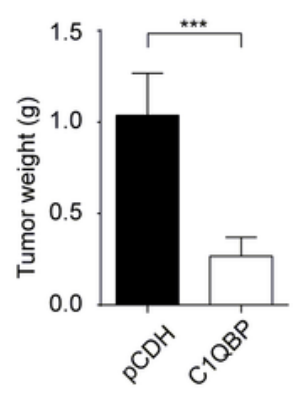

g

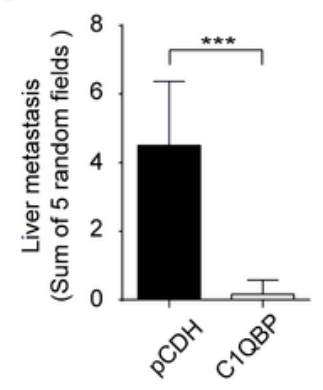

k

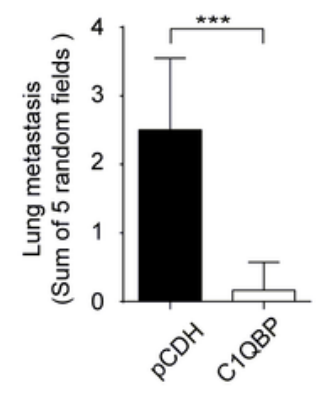

I

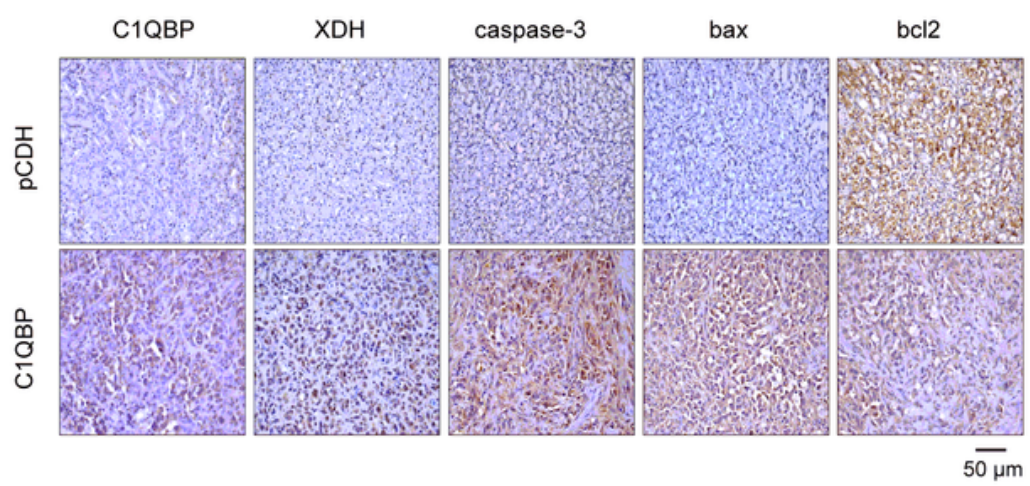

Figure 6

C1QBP suppressed RCC tumor growth, metastasis and expressions of XDH and apoptotic proteins in vivo. ACHN cells were transduced with luciferase-labeled C1QBP overexpression (C1QBP-luc) or control ( $\mathrm{pCDH}$-luc) were used to implant into BALB/c nude mice for 8 weeks, $n=6$ each group. The bioluminescence was detected by IVIS imaging system, and followed by histological examination. (a) Tumor growth was examined by IVIS imaging system, and representative images were shown. (b) 
Relative tumor bioluminescence intensity was quantified, $n=4$ each group. (c) Left primary renal tumor weight was measured and analyzed through respectively reduce corresponding weight of right renal, $\mathrm{n}=$ 6. Liver and lung metastases were examined by IVIS bioluminescence imaging system and histological H\&E staining for detection of tumor foci. Tumor metastases in liver (d) and lung ( $h$ ) were examined by IVIS imaging system, and representative images were shown. Quantified bioluminescence in liver (e) and lung (i) metastatic sites were calculated and compared, $n=4$. Tumor metastatic foci were evaluated by H\&E staining in liver (f) and lung (j), and representative images were shown, scale bar $=100 \mu \mathrm{m}$. Tumor metastatic foci in liver $(\mathrm{g})$ and lung $(\mathrm{k})$ were quantified and compared between two groups under microscope, $n=6$. Mice primary renal tumors were collected and processed for IHC staining of C1QBP, $\mathrm{XDH}$, caspase-3, bax and bcl2 and representative images were shown $(\mathrm{I})$, scale bar $=50 \mu \mathrm{m}$. All data were presented as mean $\pm S D, *, P<0.05$, ***, $P<0.001$ by Student's t-test.

\section{Supplementary Files}

This is a list of supplementary files associated with this preprint. Click to download.

- Additionalfile1.xlsx

- Additionalfile2.docx 